\title{
Staphylococcus coagulasa-negativa clínicamente significativos. Especies más frecuentes y factores de virulencia
}

\author{
Norma Fariña, Letizia Carpinelli, Margarita Samudio, Rosa Guillén, Florentina Laspina, \\ Ramona Sanabria, Sonia Abente, Ladis Rodas, Pedro González y Herminia M. de Kaspar
}

Instituto de Investigaciones en Ciencias de la Salud Asunción, Paraguay. (NF, LC, MS, RG, FL, RS, SA, HM) Laboratorio San Roque. Asunción, Paraguay $(\mathrm{LR}, \mathrm{PG})$.

Los autores declaran no tener conflicto de intereses.

Financiamiento: Fundación Hannelore-Zimmermann, Munich, Alemania.

Recibido: 19 de abril de 2013 Aceptado: 24 de julio de 2013

Correspondencia a: Norma Fariña González normafarina@gmail.com

\section{Clinically significant coagulase-negative staphylococci. Most frequent species and virulence factors}

Background: Coagulase-negative staphylococci have emerged as responsible for a large number of infections. However, it is often difficult to assess its pathogenic role or to discard it as a contaminant. Aim: The goal of this study was to identify clinically significant coagulase-negative staphylococci to the species level and their virulence factors. Isolates came from patients consulting at the San Roque Laboratory from 2009 to 2011. Material and Methods: Species identification was performed by De Paulis et al simplified method. Production of biofilm, hemolysins, lipases, lecithinases and DNase were determined by conventional methods; methicillin-resistance by diffusion method and mecA and Panton-Valentine genes, by multiplex PCR. Results: Out of 64 isolates, $40.6 \%$ were S. epidermidis; $20.3 \%$, S. haemolyticus, and $15.6 \%$, S. lugdunensis. Biofilm production was detected in $73.1 \%$ of $S$. epidermidis, $53.8 \%$ of S. haemolyticus and $40 \%$ of $S$. lugdunensis. mecA gene was identified in $69.2 \%$ of $S$. epidermidis, $92.3 \%$ of $S$. haemolyticus and none of $S$. lugdunensis. $83 \%$ of mecA (+) S. epidermidis isolates were biofilm producers as compared to $50 \%$ of the mecA (-). Conclusion: The frequency of S. lugdunensis, the most virulent coagulase-negative staphylococci species, was relatively high. The main virulence factor in $S$. epidermidis was biofilm production, being higher in those resistant to methicillin.

Key words: Coagulase-negative staphylococci -species -biofilm- mecA gene-virulence.

Palabras clave: Staphylococcus coagulasa-negativa, especie, biopelícula, gen mecA, virulencia.

\section{Introducción}

S taphylococcus coagulasa negativa (SCN) se encuentran entre los microorganismos más frecuentemente aislados en el laboratorio de microbiología. Sin embargo, su significado clínico en muchas situaciones es difícil de establecer, pues pueden ser comensales inofensivos o patógenos invasores. El protagonismo de este grupo de bacterias como patógeno ha ido en aumento y se los ha asociado con el progreso de la tecnología médica $^{1-5}$. Han sido reportados como agentes etiológicos de bacteriemias relacionadas a catéteres, peritonitis asociadas a contaminación del catéter, infecciones en válvulas derivativas ventrículo-atriales o ventrículo-peritoneales, endocarditis de válvulas protésicas y nativas, infecciones asociadas al empleo de otros dispositivos protésicos, abscesos superficiales, infecciones en piel y tejidos blandos, infecciones oftalmológicas post-quirúrgicas e infecciones urinarias $^{3,6,7}$.

Las especies más frecuentemente involucradas en patología humana son: Staphylococcus epidermidis, $S$. haemolyticus y $S$. saprophyticus que, en conjunto, alcanzan hasta $80 \%$ de los casos; el resto se debe a $S$. lugdunensis, S. hominis, S. warneri, S. simulans, S. capitis, S. auricularis, S. cohnii y otras. La patogenicidad de SCN varía entre las diferentes especies; así, Thean Yen Tan y cols., identificaron a $S$. lugdunensis como la especie más virulenta, con $91 \%$ de los aislados asociados con infecciones clínicamente significativas ${ }^{3,6,7}$.

La virulencia está fundamentalmente relacionada con la capacidad de ciertas cepas de expresar adhesinas y formar biopelículas (slime) en los dispositivos protésicos y catéteres, en cuya intimidad los microorganismos se agregan y forman macrocolonias que crecen protegidas de la acción de antimicrobianos, anticuerpos, y de otros mecanismos de defensa del hospedero. La producción de esta biopelícula es considerada un factor importante de virulencia en algunas cepas de SCN habiéndose reportado mayor dificultad en erradicar una infección crónica asociada a este fenómeno como también la frecuente asociación con una disminución de la susceptibilidad a los antimicrobianos. Además los SCN pueden sintetizar enzimas como lipasas, ADNasas, termonucleasas, hemolisinas y demás exo-enzimas que degradan los tejidos y contribuyen a la persistencia de la infección ${ }^{5,8}$.

Los SCN aislados de infecciones nosocomiales, en especial $S$. epidermidis y $S$. haemolyticus, son resistentes a múltiples microbianos, con más de $80 \%$ de resistencia a meticilina; además, $S$. haemolyticus fue el primer Staphylococcus que ha mostrado resistencia a vancomicina. 
Otros SCN han evidenciado una sensibilidad reducida a glucopéptidos. La detección de la resistencia a meticilina en $S$. epidermidis es mucho más dificultosa que en $S$. aureus ya que cepas de $S$. epidermidis con baja CIM a oxacilina pueden contener el gen mecA y ser completamente resistentes a $\beta$-lactámicos cuando se estudian en animales. La identificación del gen mecA mediante amplificación con reacción de polimerasa en cadena (RPC) o de la proteína de unión a penicilina de baja afinidad (PBP2a) codificada por el gen mecA mediante aglutinación, constituyen los métodos de detección de la resistencia a meticilina más sensibles y específicos ${ }^{9,10}$.

En Paraguay no se realiza habitualmente identificación de las diferentes especies de SCN; tampoco se confirma la resistencia a meticilina mediante la determinación del gen mecA o de la proteína PBP2a, ni existen estudios sobre virulencia de esta bacteria. Debido a la necesidad de aprender más sobre este microorganismo, llevamos a cabo este estudio cuyo objetivo fue identificar las especies más frecuentemente aisladas como agente causal de infecciones, así como sus factores de virulencia. Con este trabajo se pretende contribuir al conocimiento de un agente que se ha convertido en un patógeno de gran importancia en los últimos años y que ha sido poco estudiado.

\section{Materiales y Métodos}

Se realizó un estudio observacional, descriptivo, de corte transversal, con muestreo no probabilístico de casos consecutivos, incluyéndose en el estudio aislados identificados como SCN mediante la prueba de coagulasa en tubo, provenientes de muestras clínicas de pacientes hospitalizados o ambulatorios del Laboratorio San Roque de Asunción-Paraguay, desde marzo del año 2009 hasta julio de 2011 y que fueron considerados clínicamente significativos. Criterios empleados: aislados de hemocultivos donde hubo desarrollo de SCN con idéntico antibiotipo en dos o más muestras, urocultivos en aislamiento puro con recuento mayor a $10^{5} \mathrm{ufc} / \mathrm{ml}$ con leucocituria, líquidos biológicos purulentos y secreciones purulentas de heridas profundas en los que se observaron cocáceas grampositivas de la muestra directa y se obtuvo desarrollo monomicrobiano en la placa primaria, y puntas de catéteres centrales con recuento mayor a 100 ufc. Fueron excluidos SCN recuperados de medios de enriquecimiento.

Los aislados de SCN fueron colocados en caldo cerebro corazón conteniendo glicerol al 15\% y remitidos al Instituto de Investigaciones en Ciencias de la Salud donde fueron conservados a $-20^{\circ} \mathrm{C}$ hasta la realización de los estudios. Cada aislado remitido fue acompañado de una ficha pre-codificada de datos. Los aislados provinieron de secreciones de heridas quirúrgicas infectadas, catéter venoso central, urocultivos, hemocutivos o secreciones purulentas profundas de diversos sitios.
El protocolo de investigación fue aprobado por el Comité de Ética en Investigación (CEI) del Instituto de Investigaciones en Ciencias de la Salud, UNA.

\section{Identificación de especie}

Los SCN fueron diferenciados de otras cocáceas grampositivas catalasa positiva, como Micrococcuss spp, Planococcus spp y Rhotia spp, mediante la ausencia de halos de inhibición frente a bacitracina $(0,04 \mathrm{U})$ y por ser sensibles a furazolidona (100 U), con halos de inhibición de 15 a $35 \mathrm{~mm}$ de diámetro.

La identificación de especie se realizó utilizando el esquema simple propuesto por De Paulis y cols. ${ }^{11}$, que emplea cinco pruebas bioquímicas iniciales: pirrolidonilarilamidasa (PYR), decarboxilación de la ornitina, producción de urea, acidificación de la manosa y sensibilidad a novobiocina, y permite clasificar a los SCN en cinco grupos: Grupo S. epidermidis, Grupo S. haemolyticus, S. lugdunensis, Grupo S. saprophyticus, Grupo S. warneri y Grupo $S$. schleiferi. Además se realizaron las pruebas adicionales recomendadas por estos autores como: acidificación de trehalosa, manitol, xilosa, producción de acetoína y crecimiento en caldo tioglicolato en condiciones de anaerobiosis según método de referencia ${ }^{12}$. En los casos en que no se pudo llegar a la identificación de especie con este esquema simplificado, se utilizó el sistema comercial de identificación API-Staph (Biomerieux (C), siguiendo las instrucciones del fabricante.

En la Tabla 1 se presenta una pequeña modificación al método de De Paulis y cols., donde se muestran las pruebas consideradas de mayor utilidad para la diferenciación de las especies de SCN más frecuentes.

\section{Identificación por microsistemas de identificación API-Staph}

El API-Staph (Biomerieux(C) consiste en una tira comercial que contiene substratos deshidratados en microtubos individuales. Éstos fueron reconstituidos con el medio líquido API-Staph medium ${ }^{\circledR}$ inoculada con la bacteria a ser identificada con una turbidez 0,5 de la escala de Mac Farland. El API-Staph ${ }^{\circledR}$ incluye: producción de ácido a partir de D-glucosa, D-trehalosa, D-mannitol, D-mannosa, xilosa, maltosa, lactosa, sucrosa, $\mathrm{N}$-acetilglucosamina, raffinosa, D-fructosa, D-melibiosa, xilitol y metil-glucosamina; reducción de nitrato, fosfatasa alcalina, arginina dihidrolasa, ureasa y producción de acetoina. Los aislados identificados como $S$. saprophyticus provenientes en su totalidad de urocultivos, en número de 38 , fueron excluidos del estudio por ser un conocido uropatógeno con excelente sensibilidad a los antimicrobianos, que no constituye ningún problema y ya estudiado por varios autores, inclusive en nuestro país ${ }^{13,14}$.

\section{Factores de virulencia}

En un total de 64 aislados de SCN se determinó los 


\begin{tabular}{|c|c|c|c|c|c|c|c|}
\hline Especies & Novobiocina & Ureasa & PYR & $\begin{array}{l}\text { Ornitina } \\
\text { (MIO) }\end{array}$ & Manosa & Trehalosa & Manitol \\
\hline S. epidermidis & $\mathrm{S}$ & + & - & V & $(+)$ & - & - \\
\hline S. haemolyticus & $\mathrm{S}$ & - & + & - & - & + & V \\
\hline S. lugdunensis & $\mathrm{S}$ & V & + & + & + & + & - \\
\hline S. schleiferi subesp schleiferi & $\mathrm{S}$ & - & + & - & + & V & - \\
\hline S capitis subesp capitis & $\mathrm{S}$ & - & - & - & + & - & + \\
\hline S capitis subesp ureolyticus & $\mathrm{S}$ & + & $\mathrm{V}^{*}$ & - & + & - & + \\
\hline S. caprae & $\mathrm{S}$ & + & $\mathrm{V}^{*}$ & - & + & $(+)$ & V \\
\hline S. simulans & $S$ & + & $+*$ & - & V & V & + \\
\hline Grupo S. warneri & $\mathrm{S}$ & + & - & - & - & $+* *$ & V \\
\hline S. hominis subesp hominis & $\mathrm{S}$ & + & - & - & - & $V^{* *}$ & - \\
\hline S. hominis subesp novobiosepticum & $\mathrm{R}$ & + & - & - & - & - & - \\
\hline S. saprophyticus & $\mathrm{R}$ & + & - & - & - & + & V \\
\hline S. cohnii subesp cohnii & $\mathrm{R}$ & - & - & - & V & + & V \\
\hline S. cohnii subesp urealyticum & $\mathrm{R}$ & + & V & - & + & $+* * *$ & + \\
\hline S. xylosus & $\mathrm{R}$ & + & V & - & + & $V^{* * *}$ & + \\
\hline
\end{tabular}

siguientes factores de virulencia: producción de biopelícula, hemolisinas, lipasas, lecitinasas, ADNasas. Además, se determinó la presencia del gen mecA y la proteína PBP2a expresada por este gen, presencia del gen de la leucocidina de Panton Valentine, resistencia a meticilina por el método de difusión y la resistencia acompañante frente a 11 antimicrobianos más por el mismo método.

Producción de biopelícula: Para la estos efectos se utilizó el método de Christensen que consiste en inocular una colonia proveniente de placa de agar sangre, en tubos de $5 \mathrm{ml}$ de agar tripteina soya y luego incubar a $37^{\circ} \mathrm{C}$ por $48 \mathrm{~h}$. El contenido del tubo es removido y luego se realiza tinción con $0,25 \%$ de safranina. Una película adherente en la superficie del tubo fue tomado como evidencia de la formación de biopelícula. La ausencia de una película o la presencia de un anillo en la interfase líquido-aire se interpretó como negativo ${ }^{15}$.

Producción de hemolisina: Se determinó en placas de agar sangre cordero incubada en una atmósfera de 5\% de $\mathrm{CO}_{2}$, a $35^{\circ} \mathrm{C}$. La formación de una zona de hemólisis alrededor de las colonias aisladas se interpreta como una prueba positiva.

Producción de nucleasa: La producción de ADNasa se determinó en placas de agar ADN, se incubó durante $48 \mathrm{~h}$ a $35^{\circ} \mathrm{C}$, con el objetivo de detectar la producción de pequeñas cantidades y se reveló con ácido clorhídrico $1 \mathrm{~N}$. El aclaramiento del medio alrededor de la estría fue considerado positivo.

Producción de lipasa y lecitinasa. La actividad lipolítica se determinó en agar yema de huevo (Medio EYA), donde una zona de aclaramiento alrededor del crecimiento bacteriano se interpretó como resultado positivo para lecitinasa y una iridiscencia o brillo perlado alrededor del crecimiento bacteriano se interpretó como prueba positiva para lipasa.

Genes mecA y Panton-Valentine: La detección de los genes mecA y $p v l$ se realizó mediante una RPC múltiple simultánea, estandarizada recientemente por Carpinelli y cols., utilizando cebadores específicos descritos previamente por Murakami y cols., y Lina y cols. ${ }^{16-18}$. Los tamaños de las bandas se verificaron corriendo en paralelo un marcador de peso molecular de 100 pb (Bioline, UK) y visualizados con luz UV utilizando un transiluminador UV previa tinción con bromuro de etidio $(5 \mu \mathrm{g} / \mathrm{ml})$.

\section{Deteción de PBP2a}

Para determinar el fenotipo resistente a oxacilina se realizó detección de PBP2a con una prueba comercial basada en aglutinación de látex (Oxoid(C), siguiendo estrictamente las instrucciones del fabricante. 


\section{Prueba de susceptibilidad in vitro}

Se determinó por el método de difusión de discos en agar Mueller Hinton según recomendaciones del Clinical Laboratory Standard Institute (CLSI) ${ }^{19}$. El inóculo fue preparado utilizando una suspensión directa de colonias, para evitar que sub-poblaciones hétero-resistentes fueran opacadas por células sensibles, que crecen más rápidamente cuando se lo prepara a partir de caldo.

Para determinar la susceptibilidad a oxacilina fue utilizado el disco de cefoxitina $30 \mu \mathrm{g}$ según recomendaciones de la CLSI, como un método más preciso para predecir la resistencia a oxacilina mediada por mecA en Staphylococcus spp. Fueron considerados los siguientes puntos de corte: para SCN (distinto de S. lugdunensis), halos de inhibición $\geq 25 \mathrm{~mm}$ fueron considerados sensibles a oxacilina y halos $\leq 24 \mathrm{~mm}$ como resistentes y para S. lugdunensis: $\geq 22 \mathrm{~mm}$ sensible a oxacilina $\mathrm{y} \leq 21 \mathrm{~mm}$, resistentes.

También se determinó la susceptibilidad in vitro a penicilina, eritromicina, clindamicina, gentamicina, ciprofloxacina, moxifloxacina, rifampicina, cotrimoxazol, tetraciclina, cloranfenicol y vancomicina por el método de difusión de discos en agar Mueller Hinton según recomendaciones del $\mathrm{CLSI}^{19}$. Se evaluó además la resistencia inducible a clindamicina mediante el $\mathrm{D}$ test.

\section{Cepas de referencia}

Fueron utilizadas cepas de referencia de la American Type Culture Collection (ATCC) S. haemolyticus: ATCC 29970, S. lugdunensis: ATCC 49576 y S. aureus: ATCC 25923 para control de las pruebas de identificación y los factores de virulencia. Para determinación de sensibilidad por difusión fue utilizado $S$. aureus: ATCC 25923 y para control de los métodos moleculares $S$. aureus: ATCC 25923 y S. aureus: ATCC 43300 (Microbiologics ${ }^{\circ}$ ).

\section{Resultados}

Se estudió un total de 64 aislados de SCN provenientes de: urocultivos (n: 22), secreciones purulentas profundas de diversas localizaciones (n: 14), secreciones de heridas infectadas (n: 9), catéter venoso central (n: 8), abscesos (n: 4), líquido peritoneal (n: 2), hemocultivos (n: 2) y hueso (n: 2). Estas muestras fueron de pacientes internados en $42,2 \%$ de los casos.

La especie más frecuentemente aislada fue $S$. epidermidis 26 (40,6\%), seguida de S. haemolyticus 13 (20,3\%), y S. lugdunensis $10(15,6 \%)$ (Tabla 2).

Considerando el origen de las distintas especies de SCN se obtuvieron de orina aislados de: S. epidermidis $10(45,5 \%)$, S. haemolyticus $6(27,3 \%)$ y $S$. capitis subesp. capitis $3(13,6 \%)$; de secreciones purulentas de diversas localizaciones, abscesos, heridas: S. lugdunensis
$10(37,0 \%)$, S. epidermidis $7(25,9 \%)$ y $S$. haemolyticus $5(18,5 \%)$; y de hemocultivos, catéteres y huesos: $S$. epidermidis 9 (60\%), S. haemolyticus $2(13,3 \%)$ y $S$. capitis subesp. capitis $2(13,3 \%)$. Todos los aislados de S. lugdunensis fueron a partir de secreciones purulentas, abscesos y heridas (Tabla 3).

Las muestras de orina provinieron en su mayoría de pacientes ambulatorios. En 86,4\%, las de secreciones purulentas de diversas localizaciones, abscesos, heridas, fueron también mayoritariamente de ambulatorios en $63,0 \%$, a diferencia de muestras extraídas de sangre, catéteres y hueso, grupo de muestras que provino fundamentalmente de pacientes hospitalizados en $93,3 \%$ (Tabla 4).

La edad media de los pacientes con urocultivo fue de 78,4 años, mientras que la edad media de los demás pacientes fue de 54 años, observándose una diferencia estadísticamente significativa.

Tabla 2. Frecuencia de aislamiento de las distintas especies de Staphylococcus coagulasa negativa

\begin{tabular}{lcc|} 
Especie aislada & Frecuencia & $\%$ \\
S. epidermidis & 26 & 40,6 \\
S. haemolyticus & 13 & 20,3 \\
S. lugdunensis & 10 & 15,6 \\
\hline S. capitis. capitis & 5 & 7,8 \\
S. warneri & 3 & 4,7 \\
S. caprae & 2 & 3,1 \\
S. simulans & 2 & 3,1 \\
S. xylosus & 2 & 3,1 \\
S. hominis sub esp. hominis & 1 & 1,6 \\
Total & 64 & 100,0 \\
\hline
\end{tabular}

Tabla 3. Frecuencia de las diferentes especies aisladas según origen de la muestra

\begin{tabular}{|c|c|c|c|c|}
\hline & Orina & $\begin{array}{l}\text { Secreciones purulentas, } \\
\text { abscesos, heridas }\end{array}$ & $\begin{array}{l}\text { Hemocultivos, punta } \\
\text { de catéteres, hueso }\end{array}$ & Total \\
\hline S. epidermidis & $10(45,5 \%)$ & $7(25,9 \%)$ & $9(60,0 \%)$ & 26 \\
\hline S. haemolyticus & $6(27,3 \%)$ & $5(18,5 \%)$ & $2(13,3 \%)$ & 13 \\
\hline S. lugdunensis & 0 & $10(37,0 \%)$ & 0 & 10 \\
\hline S. capitis & $3(13,6)$ & 0 & $2(13,3 \%)$ & 5 \\
\hline S. warneri & $1 \quad(4,5 \%)$ & $1 \quad(3,7 \%)$ & $1 \quad(6,7 \%)$ & 3 \\
\hline S. caprae & $1 \quad(4,5 \%)$ & $1 \quad(3,7 \%)$ & 0 & 2 \\
\hline S. simulans & 0 & $2(7,4 \%)$ & 0 & 2 \\
\hline S. xylosus & $1 \quad(4,5 \%)$ & $1 \quad(3,7 \%)$ & 0 & 2 \\
\hline S. hominis & 0 & 0 & $1 \quad(6,7 \%)$ & 1 \\
\hline Total & 22 & 27 & 15 & 64 \\
\hline
\end{tabular}


Tabla 4. Distribución de las muestras analizadas por procedencia del paciente

\begin{tabular}{lccc} 
Muestras & \multicolumn{2}{c}{$\begin{array}{c}\text { Procedencia de los pacientes } \\
\text { Ambulatorio }\end{array}$} & Internado \\
Orina & $19(86,4)$ & $3(13,6)$ & 22 \\
Secreciones purulentas, abscesos, secreciones de heridas & $17(63,0)$ & $10(37,0)$ & 27 \\
Hemocultivo, punta de catéteres, hueso & $1(6,7)$ & $14(93,3)$ & 15 \\
Total & $37(57,8)$ & $27(42,2)$ & 64 \\
\hline
\end{tabular}

Tabla 5. Frecuencia de los factores de virulencia en las especies principales de Staphylococcus coagulasa negativa

$\begin{array}{lccc}\text { Factores de virulencia } & \begin{array}{c}\text { S. epidermidis } \\ (\mathrm{n}=26)\end{array} & \begin{array}{c}\text { Especie aislada } \\ \text { S. haemolyticus } \\ (\mathrm{n}=13)\end{array} & \begin{array}{c}\text { S. lugdunensis } \\ (\mathrm{n}=10)\end{array} \\ \text { Biopelícula } & 19(73,1) & 7(53,8) & 4(40) \\ \text { Lipasa } & 14(53,8) & 8(61,5) & 6(60) \\ \text { Lecitinasa } & 14(53,8) & 6(46,2) & 0 \quad(0) \\ \text { ADNasa* } & 0(0) & 13(100) & 10(100) \\ \text { Hemolisina } & 4(15,4) & 13(100) & 10(100) \\ \text { Mec A } & 18(69,2) & 12(92,3) & 0 \quad(0) \\ \text { PVL } & 0(0) & 0(0) & 0 \quad(0)\end{array}$

Los valores entre paréntesis expresan porcentajes. *Prueba de ADNasa con 48 h de incubación PVL: leucocidina Panton Valentine.

Tabla 6. Resistencia a los diferentes antimicrobianos ensayados de las principales especies de Staphylococcus coagulasa negativa

\begin{tabular}{|lccc|}
\hline Antimicrobiano & $\begin{array}{c}\text { S. epidermidis } \\
(\mathrm{n}=26)\end{array}$ & $\begin{array}{c}\text { Especie aislada } \\
\text { S. haemolyticus } \\
(\mathrm{n}=13)\end{array}$ & $\begin{array}{c}\text { S. Iugdunensis } \\
(\mathrm{n}=10)\end{array}$ \\
Penicilina & $25(96,2)$ & $12(92,3)$ & $3(30)$ \\
Oxacilina & $17(65,4)$ & $12(92,3)$ & $0(0)$ \\
Clindamicina* & $8(30,8)$ & $5(38,5)$ & $1(10)$ \\
Eritromicina & $14(53,8)$ & $11(84,6)$ & $1(10)$ \\
Cotrimoxazol & $8(30,8)$ & $9(69,2)$ & $0(0)$ \\
Ciprofloxacina & $20(76,9)$ & $10(76,9)$ & $0(0)$ \\
Moxifloxacina & $14(53,8)$ & $9(69,2)$ & $0(0)$ \\
Cloranfenicol & $7(26,9)$ & $4(30,8)$ & $0(0)$ \\
Gentamicina & $6(23,1)$ & $9(69,2)$ & $0(0)$ \\
Rifampicina & $7(26,9)$ & $7(53,8)$ & $1(10)$ \\
Tetraciclina & $2(7,7)$ & $1(7,7)$ & $0(0)$ \\
\hline Los valores entre paréntesis expresan porcentajes. * ${ }^{*}$ La resistencia a clindamicina incluye resistencia \\
inducible.
\end{tabular}

Tabla 7. Frecuencia de producción de biopelícula en las especies principales de Staphylococcus coagulasa negativa, según presencia mecA

\begin{tabular}{lccc} 
& \multicolumn{3}{c}{ Producción de biopelícula } \\
& mecA (+) & mecA (-) \\
S. epidermidis & $15 / 18(83,3 \%)$ & $4 / 8 \quad(50 \%)$ \\
S. haemolyticus & $7 / 12(58,3 \%)$ & $0 / 1 \quad(0 \%)$ \\
S. lugdunensis & $0 / 0(0 \%)$ & $4 / 10(40 \%)$ \\
\hline
\end{tabular}

E1 73,1\% de S. epidermidis, 53,8\% de S. haemolyticus y $40 \%$ de $S$. lugdunensis fueron productores de biopelícula (Tabla 5).

La producción de lipasa y lecitinasa fue de $53,8 \%$ para $S$. epidermidis y $S$. haemolyticus de 61,5 y $46,2 \%$, respectivamente. $S$. lugdunensis sólo fue positiva para la producción de lipasa en $60 \%$ y ningún aislado produjo lecitinasa (Tabla 5).

Nucleasa de tipo ADNasa se detectó en $100 \%$ de las cepas de $S$. haemolyticus y de $S$. lugdunensis con la incubación durante $48 \mathrm{~h}$. La producción fue menor en relación a la producida por la cepa de $S$. aureus ATCC 25923. Ningún aislado de $S$. epidermidis fue productor de ADNasa (Tabla 5). Cuando la incubación de la prueba de ADNasa es realizada durante $24 \mathrm{~h}$ como lo describe la técnica para identificación de especies, tanto $S$. haemolyticus como S. lugdunensis dan prueba de ADNasa negativa.

La producción de hemolisina se observó en todos los aislados de S. haemolyticus y de S. lugdunensis, siendo mucho más intensa la hemólisis producida por el primero (Tabla 5).

Los resultados del gen mecA coincidieron totalmente con la presencia de PBP2a y fue de $69,2 \%$ en $S$. epidermidis, $92,3 \%$ en $S$. haemolyticus y en ninguno de los aislados de $S$. lugdunensis (Tabla 5).

No se detectó el gen de la PVL en aislado alguno de SCN.

Los aislados de $S$. epidermidis y $S$. haemolyticus presentaron una alta resistencia a la mayoría de los antimicrobianos ensayados; en cambio, para S. lugdunensis se obtuvo muy buena sensibilidad (Tabla 6).

Los valores de resistencia a clindamicina obtenidos incluyen las resistencias inducibles, fenómeno que se observó en dos aislados de $S$. epidermidis, uno de $S$. haemolyticus y en el único aislado de S. lugdunensis resistente a clindamicina. Además fue observado en un aislado de $S$. simulans.

Por el método de difusión utilizando el disco de cefoxitina, la resistencia a meticilina fue de $65,4 \%$ en $S$. epidermidis y $92,3 \%$ en $S$. haemolyticus. Ningún aislado de $S$. lugdunensis mostró resistencia a meticilina.

De los 30 aislados mecA (+), 29 (96,7\%) fueron detectado por test de difusión.

El $83 \%$ de $S$. epidermidis gen mecA positivos fue productor de biopelícula (Tabla 7).

\section{Discusión}

Nuestros hallazgos coinciden con numerosos trabajos que reportan a $S$. epidermidis como el SCN más frecuentemente implicado en infecciones, seguido por S. haemolyticus ${ }^{11,20,21}$. S. lugdunensis fue identificado en $15 \%$ de las cepas, frecuencia que consideramos alta 
cuando la comparamos con lo reportado por estos mismos autores, de 3 a $6 \%$. Esta especie está reportándose cada vez con mayor frecuencia y se la ha asociado a un amplio espectro de infecciones, principalmente de piel y tejidos blandos, como celulitis y abscesos subcutáneos. Además, ha sido reportada como causa de endocarditis, artritis, infecciones de prótesis, osteomielitis, infección urinaria y de heridas, en los que se describe la naturaleza agresiva de esta bacteria, considerada más virulenta que otros $\mathrm{SCN}^{22,23}$. El incremento de infecciones debidas a $S$. lugdunensis puede deberse tanto a un mejor conocimiento de sus características, o a un mayor índice de sospecha; sin embargo, es posible que su incidencia siga aún subestimada e incluida dentro del grupo de los SCN no identificados, o identificado como $S$. aureus, debido a que comparte con este último, la capacidad de producir coagulasa ligada y la similitud de sus colonias. En frecuencia de aislamiento sigue $S$. capitis subesp. capitis, frecuencia comparable a lo encontrado por otros autores, quienes lo reportan como causante de infecciones varias, abscesos y endocarditis ${ }^{24,25}$. Como era de esperarse, las demás especies, S. warneri, S. caprae, S. hominis subespecie hominis y $S$. simulans, fueron aisladas en baja frecuencia; existen, en cambio, reportes que revelan un aumento de $S$. hominis y $S$. warneri como causantes de bacteriemia e infecciones nosocomiales ${ }^{7,26}$. No se obtuvo aislamiento de $S$. schleiferi, que, si bien no es frecuente, comparte con S. lugdunensis la capacidad de producir coagulasa unida e infecciones similares a las de $S$. aureus ${ }^{27}$.

En este estudio se excluyeron a los aislados de $S$. saprophyticus, conocido uropatógeno, frecuentemente aislado en mujeres sexualmente activas y que ya ha sido bien estudiado ${ }^{1,13,14}$. Esta especie es identificada en los laboratorios de microbiología mediante la prueba de resistencia a la novobiocina, prueba considerada suficiente para la identificación siempre que el aislado provenga de orina de mujeres en edad sexual activa. Otros SCN resistentes a novobiocina son rarísimos como agentes de infección del tracto urinario en este grupo etario. Obtuvimos solamente dos aislados resistentes a novobiocina que no correspondieron a $S$. saprophyticus, por lo que no fueron excluidos del estudio; ambos fueron identificados como S. xylosus, uno de ellos de orina de un hombre añoso y el otro de secreción de herida. A pesar de que los aislados de S. saprophyticus fueron excluidos en este trabajo, 34,4\% de los aislados se obtuvieron de muestras de orina, comparable con el estudio de De Paulis y cols., para quienes el porcentaje de muestras de orina fue similar al nuestro, pero sin la exclusión de $S$. saprophyticus. En dicho estudio, siguieron en frecuencia las muestras de heridas $(14,9 \%)$ y luego hemocultivos (12,9\%). En otras series predominan totalmente muestras de hemocultivos, como en el reporte de Ieven y cols., quienes obtuvieron la mayor parte de aislados de hemocultivos en $60,3 \%$ de los casos, seguido de catéteres (12,1\%), luego heridas (10,8\%), y la orina representó sólo $5,6 \%$ de las muestras ${ }^{11,20}$. El número importante de orinas en nuestro estudio, podría deberse a que el laboratorio donde fueron recolectadas las muestras recibe una mayor cantidad de pacientes ambulatorios y la mayoría de los urocultivos procedió de pacientes no hospitalizados con edad media significativamente mayor a la edad media de los demás pacientes. Las razones posibles de la elevada frecuencia de infecciones del tracto urinario en los ancianos incluyen uropatía obstructiva debido a hiperplasia prostática y pérdida de la actividad bactericida de las secreciones prostáticas en los varones, vaciamiento deficiente de la vejiga debido al prolapso en las mujeres, además enfermedad neuromuscular, aumento de la manipulación y uso de catéteres en la vejiga de ambos $\operatorname{sexos}^{28}$. Además, existen reportes de pacientes con prostatitis subaguda o crónica debidas a $\mathrm{SCN}^{29}$. De los SCN distintos de S. saprophyticus cultivados a partir de orina, $S$. epidermidis es la especie predominante y aislado de pacientes con complicaciones de las vías urinarias $^{30}$. Coincidentemente, en el presente estudio el más frecuentemente aislado de muestras de orina fue $S$. epidermidis, seguido de $S$. haemolyticus.

La totalidad de los aislados de $S$. lugdunensis provino de secreciones purulentas de diversos orígenes, de abscesos y de heridas; esta especie, como ya se ha mencionado, es aislada principalmente de piel y tejidos blandos ${ }^{22,23}$. No se identificó cepa alguna de $S$. lugdunensis a partir de orina.

En cuanto al método utilizado para la identificación de especies, es económico, simple como lo manifiestan los autores en relación al método de referencia propuesto por Kloss y cols. ${ }^{12}$, y además efectivo porque permitió la identificación de las especies más frecuentes con relativa facilidad.

Es importante mencionar que en países en vías de desarrollo como el nuestro, la identificación del género Staphylococcus se realiza en la mayoría de los laboratorios de microbiología, con la prueba de coagulasa en tubo; si ésta es positiva se informa como $S$. aureus y si resulta negativa se identifica como $\mathrm{SCN}^{21}$. Sin embargo, debido al aumento de las infecciones debidas a estos agentes se los debería identificar a nivel de especie, por lo menos a las más frecuentemente asociadas con patologías. Coincidimos con numerosos trabajos que han enfatizado la importancia de realizar identificación de especie en todo aislado que se considere significativo ${ }^{7}$, por lo que se propone la implementación del método simple y económico, que utiliza medios con lo que habitualmente cuentan los laboratorios de microbiología y que permite diferenciar las especies más frecuentemente implicadas en infecciones como son $S$. epidermidis, S. haemolyticus, $S$. lugdunensis e inclusive, $S$. capitis sub especie capitis.

Las demás especies como $S$. hominis, $S$. warneri, $S$. 
simulans y $S$. capitis subsp. ureolyticus son más difíciles de identificar por esquemas simples, pues dan variables a algunas de las pruebas y muchas veces son necesarias otras pruebas suplementarias. En nuestro trabajo utilizamos el microsistema API para la confirmación de estas especies. Se han reportado errores de identificación, principalmente en estas especies, con muchos de los métodos, inclusive los automatizados como Vitek ${ }^{\circledR}$ (bioMérieux), cuando se lo compara con métodos moleculares ${ }^{21}$. Se reporta muchos errores de identificación de $S$. lugdunensis con equipos automatizados o sistemas comerciales que aún no incorporan la prueba de la ornitina que es una prueba clave. Hemos propuesto un esquema para la identificación de las especies más frecuentes de $\mathrm{SCN}$, utilizando el método de De Paulis y cols. ${ }^{11}$, con pequeñas modificaciones, donde se incluyeron además de las cinco pruebas iniciales (resistencia a novobiocina, producción de ureasa, hidrólisis de pirrolidonilarilamidasa, acidificación de manosa y ornitina decarboxilasa), dos pruebas que los mencionados autores citan como adicionales: la acidificación de trehalosa y manitol, porque consideramos pruebas de gran utilidad para diferenciar en una sola etapa a $S$. epidermidis, especie más frecuentemente aislada, de $S$. capitis sub especie ureolyticus y $S$. caprae, ya que $S$. epidermidis arroja resultado negativo para ambas pruebas, en cambio $S$. capitis sub especie ureolyticus es manitol positivo y trehalosa negativa, mientras $S$. caprae es positiva lenta para trehalosa y variable para manitol. Esquemas simplificados de identificación como el de Ieven y cols., e Iorio y cols., incluyen la acidificación de la trehalosa entre las pruebas iniciales ${ }^{20,21}$.

Hemos demostrado, como otros autores, que SCN posee varios factores de virulencia ${ }^{1,5,8}$. Encontramos que la producción de biopelícula es uno de los más importantes, especialmente en $S$. epidermidis, causante de infecciones de implantes de dispositivos médicos, lo cual está directamente relacionado a la capacidad que posee de expresar adhesinas y formar multicapas de biopelícula en cuerpos extraños como superficies lisas de plástico, catéteres, válvulas cardiacas protésicas y marcapasos ${ }^{31,32}$. En nuestro estudio obtuvimos valores considerables de producción de biopelícula, a diferencia de lo encontrado por Ribeiro da Cunha y cols.: 20 (17,1\%) en 117 aislados de recién nacidos. En dicho estudio se tomaron aislados contaminantes y significativos; no hubo diferencias significativas cuando separaron los dos grupos ${ }^{5}$. En cambio, Kitao y cols., en un reciente estudio investigando el gen ica relacionado con la producción de biopelícula y el gen mecA en $S$. epidermidis provenientes de hemocultivos y de microbiota nasal y de mano, encontraron una diferencia muy importante entre la producción de biopelícula entre los aislados de hemocultivos y los de microbiota normal ${ }^{33}$.

Se observó también una importante producción de lipasa en todas las especies. En este estudio todos los aislados fueron positivos, por lo menos, para un factor de virulencia estudiado, presumiblemente porque los aislados fueron a partir de procesos infecciosos. No se detectó cepa alguna que fuera positiva para el gen de la leucocidina de Panton-Valentine, factor de virulencia frecuente en cepas de $S$. aureus aislados de infecciones adquiridas en la comunidad ${ }^{34}$. Si bien la prueba de ADNasa, utilizada para la identificación de especies de Staphylococcus es negativa para S. lugdunensis como para S. haemolyticus con incubación de $24 \mathrm{~h}$, al estudiar ADNasa como un factor de virulencia, con una incubación prolongada de $48 \mathrm{~h}$, se encontró que todos los aislados, tanto de S. lugdunensis como de $S$. haemolyticcus, produjeron ADNasa, observándose una producción menor con respecto a $S$. aureus.

A pesar de que la resistencia bacteriana no es considerada como un factor de virulencia propiamente dicho, lo incluimos en el estudio ya que es una característica emergente y preocupante, especialmente en $S$. epidermidis y $S$. haemolyticcus. Se ha visto que en general las cepas que poseen gen mecA producen biopelícula en mayor cantidad $^{33}$. En nuestro estudio, $83 \%$ de las cepas de $S$. epidermidis mecA positivo resultaron productores de biopelícula. Este hecho complica el tratamiento por la mayor dificultad en erradicar una infección crónica asociada a la producción de biopelícula; más aún en el paciente con dispositivos protésicos y catéteres en cuya intimidad los microorganismos se agregan y forman macrocolonias que crecen protegidas de la acción de antimicrobianos, anticuerpos, y del resto de los mecanismos de defensa del hospedero ${ }^{5,8}$.

Hasta el presente se utilizan las terapias convencionales con agentes antimicrobianos para tratar y prevenir las infecciones asociadas a estos dispositivos. Sin embargo, el tratamiento de estas infecciones es muy difícil ya que la bacteria dentro de la biopelícula y la superficie del cuerpo extraño frecuentemente sobrevive y la remoción del dispositivo es casi siempre requerida.

Coincidente con numerosos trabajos, la resistencia a meticilina observada en $S$. epidermidis y $S$. haemolyticus fue muy alta. Pudimos observar una marcada resistencia acompañante, especialmente a ciprofloxacina en ambas especies y además a eritromicina en S. haemolyticus. Tetraciclina mostró buena actividad in vitro para las dos especies.

En este estudio se ha evaluado vancomicina por el método de difusión mostrando $100 \%$ de actividad in vitro si se considera los puntos de corte de la CLSI utilizados hasta el 2008. Actualmente ya no se aconseja el método de difusión y debe realizarse la determinación de la CIM a vancomicina. Existen reportes de aislados de $S$. haemolyticus y $S$. epidermidis resistentes a vancomicina y otros glucopéptidos ${ }^{7}$.

En cuanto a los métodos utilizados para la detección de la resistencia a meticilina, se obtuvo excelente correlación 
entre los métodos de detección del gen mecA por RPC y el comercial de aglutinación que detecta la proteína ligadora de penicilina de baja afinidad, la PBP 2a.

Además, se obtuvo muy buena correlación entre el método de difusión y la presencia del gen mecA; sólo un aislado de $S$. epidermidis que presentaba el gen no fue detectado por el método de difusión con los discos de cefoxitina según puntos de corte propuesto por el CLSI ${ }^{19}$. Ha sido descrito para S. epidermidis que algunos aislados, aún portando el gen, tienen CIM relativamente bajas lo que no puede ser detectado por difusión.

Por otra parte, se debe destacar la excelente sensibilidad que presenta $S$. lugdunensis a la mayoría de los antimicrobianos. No encontramos resistencia a meticilina en concordancia con numerosos estudios que la consideran una bacteria muy sensible, característica que comparte con S. saprophyticus. Sin embargo, existen reportes de aislados de $S$. lugdunensis resistentes a meticilina ${ }^{35}$.

Con este estudio contribuimos al conocimiento de un patógeno emergente que puede causar enfermedades graves, muchas veces difícil de erradicar. Proponemos que la identificación a nivel de especie sea una práctica habitual en los laboratorios de rutina de microbiología clínica, incluso los que no cuentan con equipos automatizados de identificación, para ayudar a valorar a esta bacteria en su justa medida.

No debe olvidarse que el tratamiento de $S$. epidermidis y $S$. haemolyticcus, especialmente relacionadas a dispositivos ajenos, es un desafío para la ciencia médica por la producción de biopelícula y la resistencia antimicrobiana, por lo que se propone seguir estudiando en este tema, a fin de contribuir con investigaciones que se llevan a cabo actualmente y que están dirigidas a interferir en la formación de biopelícula o posibilitar la disgregación del mismo.
Agradecimientos: A la Fundación Georg-Hannelore Zimmermann, Munich, Alemania, por el apoyo económico para la realización del proyecto.

\section{Resumen}

Introducción: Staphylococcus coagulasa-negativa ha emergido como responsable de un gran número de infecciones. No obstante, con frecuencia es difícil asegurar su rol patógeno o descartarlo como contaminante. Objetivo: Estudiar a nivel de especies Staphylococcus coagulasa-negativa clínicamente significativos y sus factores de virulencia, de aislados provenientes de pacientes del Laboratorio San Roque de Asunción, Paraguay entre los años 2009 y 2011. Material y Métodos: Para la identificación de especies fue utilizado el método simplificado de De Paulis y cols. La producción de biopelícula, hemolisinas, lipasas, lecitinasas, ADNasa, fue determinada por métodos convencionales; la resistencia a meticilina por difusión y los genes mecA y Panton-Valentine por RPC múltiple. Resultados: De 64 aislados, 40,6\% correspondió a $S$. epidermidis, 20,3\% $S$. haemolyticus y $15,6 \% \mathrm{~S}$. lugdunensis. La producción de biopelícula fue detectada en S. epidermidis en 73,1\%, S. haemolyticus 53,8\% y S. lugdunensis 40\%. El gen mecA fue identificado en $69,2 \%$ de $S$. epidermidis, $92,3 \%$ de $S$. haemolyticus y en ninguno de S. lugdunensis. El 83\% de $S$. epidermidis mecA (+) fue productor de biopelícula en comparación a 50\% de los mecA (-). Conclusión: La frecuencia de $S$. lugdunensis, una de las especies más virulentas de Staphylococcus coagulasa-negativa fue relativamente alta; y el principal factor de virulencia en $S$. epidermidis fue la producción de biopelícula, siendo mayor en los resistentes a meticilina.

\section{Referencias bibliográficas}

1.- Koneman E, Allen S, Janda W, Schreckenberger P, Winn W. Diagnóstico microbiológico. Texto y Atlas a color. 5ta ed. Buenos Aires: Médica Panamericana 1999; p. 282-3.

2.- Kloos W E, Schleifer K H. Isolation and characterization of staphylococci from human skin. Descriptions of four new species: Staphylococcus warneri, Staphylococcus capitis, Staphylococcus hominis, and Staphylococcus simulans. Int J Syst Bacteriol 1975; 25: 62-79.

3.- $\quad$ Tan T Y, Ng S Y, Ng W X. Clinical significance of coagulase negative staphylococci recovered from nonsterile sites. J Clin Microbiol 2006; 44: 3413-4.

4.- Aldea-Mansilla C, García de Viedma D,
Cercenado E, Martín-Rabadán P, Marín M, Bouza E. Comparison of phenotypic with genotypic procedures for confirmation of coagulase-negative Staphylococcus catheterrelated bloodstream infections. J Clin Microbiol 2006; 44: 3529-32.

5.- Cunha M L, Rugolo L M, Lopes C A. Study of virulence factors in coagulase-negative staphylococci isolated from newborns. Mem Inst Oswaldo Cruz 2006; 101: 661-8.

6.- Predari S. Estafilococos coagulasa negativos: el enemigo silente. Rev Arg Microbiol 2007; 39: 1-3.

7.- Kloos W E, Bannerman T L. Update on clinical significance of coagulase-negative staphylococci. Clin Microbiol Rev 1994; 7 : 117-40.

8.- Arslan S, Özkardes F. Slime production and antibiotic susceptibility in staphylococci isolated from clinical simples. Mem Inst Oswaldo Cruz 2007; 102: 29-33.

9.- Archer G L, Climo M W. Staphylococcus epidermidis y otros estafilococos coagulasa negativos. En Mandell G L, Douglas R G, Bennett J E (eds). Enfermedades Infecciosas. Principios y Práctica. $6^{\mathrm{a}}$ ed. Madrid: Elsevier; 2006. p. 2352-60.

10.- Corso A, Soloaga R, Faccone D, Gagetti P, Corbella S, Iglesias M, et al. Improvement of a latex agglutination test for the evaluation of oxacillin resistance in coagulase negative staphylococci. Diagn Microbiol Infect Dis 2004; 50: 223-5.

11.- De Paulis A, Predari S, Chazarreta C, Santoianni J. Five-test simple scheme for species-level identification of clinical significant coagulase-negative staphylococci. J Clin Microbiol 2003; 41: 1219-24. 
12.- Kloos W E, Schleifer K H. 1975. Simplified scheme for routine identification of human Staphylococcus species. J Clin Microbiol 1975; 1: 82-8.

13.- Pead L, Maskell R, Morris J. Staphylococcus saprophyticus as a urinary pathogen: a six year prospective survey. Br Med J 1985; 291 : 1157-9.

14.- Fariña N, Sanabria R, Ramos L, Samudio M, Figueredo L. Staphylococcus saprophyticus como patógeno urinario. Mem Inst Invest Cienc Salud 2005; 1: 31-3.

15.- Christensen G D, Simpson W A, Bisno A L, Beachey E H. Adherence of slime producing strains of $S$. epidermidis to smooth surfaces. Infect Immun 1982; 37: 318-26.

16.- Murakami K, Minamide W, Wada K, Nakamura E, Teraoka H, Watanabe S. Identification of methicillin-resistant strains of staphylococci by polymerase chain reaction. J Clin Microbiol 1991; 29: 2240-4.

17.- Lina G, Piémont Y, Godail-Gamot F, Bes M, Peter M, Gauduchon V, et al. Involvement of Panton-Valentine leukocidin-producing Staphylococcus aureus in primary skin infections and pneumonia. Clin Infect Dis 1999; 29: 1128-32.

18.- Carpinelli L M, Guillén R M, Fariña N, Basualdo W, Aquino R. PCR múltiple para la detección simultánea de los genes mecA y $p v l$ en Staphylococcus spp Mem Inst Investig Cienc Salud 2012: 10 (1): 5-13.

19.- Clinical and Laboratory Standards Institute. Performance standards for antimicrobial susceptibility testing; nineteenth informational supplement and approved standard M100-S19. Wayne, PA: CLSI; 2009.

20.- Ieven M, Verhoeven J, Pattyn S, Goossens H. Rapid and economical method for species identification of clinically significant coagulasenegative staphylococci. J Clin Microbiol 1995; 33: 1060-3.

21.- Iorio N L, Ferreira R B, Schuenck R P, Malvar K L, Brilhante A P, Nunes AP, et al. Simplified and reliable scheme for specieslevel identification of Staphylococcus clinical isolates. J Clin Microbiol 2007; 45: 2564-9.

22.- Cercenado E. Staphylococcus lugdunensis: un estafilococo coagulasa negativo diferente de los demás. Enferm Infecc Microbiol Clin 2009; 27 (3): 139-42.

23.- Mateo M, Maestre J R, Aguilar L, Cafini F, Puente P, Sánchez P, et al. Genotypic versus phenotypic characterization, with respect to susceptibility and identification, of 17 clinical isolates of Staphylococcus lugdunensis. J Antimicrob Chemother 2005; 56: 287-91.

24.- Surani S, Chandna H, Weinstein R. 1993. Breast abscess: coagulase-negative staphylococcus as a sole pathogen. Clin Infect Dis 1993; 17: 701-4.

25.- Mainardi J L, Lortholary O, Buu-Hoi A, Desplaces H, Goldstein F, Gutmann L, et al. Native valve endocarditis caused by Staphylococcus capitis. Eur J Clin Microbiol Infect Dis 1993; 12: 789-91.

26.- Kamath U, Singer C, Isenberg D. Clinical significance of Staphylococcus warneri bacteremia. J Clin Microbiol 1992; 30: 261-4.

27.- Hébert G A. Hemolysins and other characteristics that help differentiate and biotype
Staphylococcus lugdunensis and Staphylococcus schleiferi. J Clin Microbiol 1990; 28: 2425-31.

28.- Baldassarre J S, Kaye D. Special problems of urinary tract infection in the elderly. Med Clin North Am 1991; 75: 375-90.

29.- Nickel J C, Costerton J W. Coagulase-negative staphylococcus in chronic prostatitis. J Urol 1992; 147: 398-401.

30.- Nicolle L E, Hoban S A, Harding G K M. Characterization of coagulase-negative staphylococci from urinary tract specimens. J Clin Microbiol 1983; 17: 267-71.

31.- Vogel L, Sloos J H, Spaargaren J, Suiker I, Dijkshoom L. Biofilm production by Staphylococcus epidermidis isolates associated with catheter related bacteremia. Diagn Microbiol Infect Dis 2000; 36: 139-41.

32.- McCann M T, Gilmore B F, Gorman S P. Staphylococcus epidermidis device-related infections: pathogenesis and clinical management. JPP 2008; 60: 1551-71.

33.- Kitao T, Ishimaru M, Nishihara S. Detection of biofilm-producing and methicillin resistance genes in Staphylococcus epidermidis isolated from healthy humans and in blood culture tests. J Infect Chemother 2010; 16: 170-3.

34.- Kaplan S. Community-acquired methicillinresistant Staphylococcus aureus infections in children. Semin Pediatr Infect Dis 2006; 17: 113-9.

35.- Tee W S, Soh S Y, Lin R, Loo L H. Staphylococcus lugdunensis carrying the mecA gene causes catheter-associated blood stream infection in premature neonate. J Clin Microbiol 2003; 41: 519-20. 